\title{
Crescimento populacional de Moina sp. alimentadas com diferentes dietas para uso na larvicultura e aquariofilia
}

\author{
Population growth of Moina sp. fed with different diets for use in larviculture and aquarium \\ farming \\ Crecimiento de la población de Moina sp. alimentadas con diferentes dietas para su uso en \\ larvicultura y cultivo de acuarios
}

Recebido: 22/09/2021 | Revisado: 29/09/2021 | Aceito: 03/10/2021 | Publicado: 04/10/2021

\author{
Katiani Chaves Rocha \\ ORCID: https://orcid.org/0000-0001-8398-8269 \\ Universidade Federal do Piauí, Brasil \\ E-mail: katianichaves189@gmail.com \\ Luis Ricardo Romero Arauco \\ ORCID: https://orcid.org/0000-0003-3454-835X \\ Universidade Federal do Piauí, Brasil \\ E-mail: luisricardora@gmail.com \\ Arléia Medeiros Maia \\ ORCID: https://orcid.org/0000-0002-3674-973X \\ Rivelli Alimentos S.A, Brasil \\ E-mail: arleiamederiosmaia@gmail.com \\ José Luiz Leonardo de Araujo Pimenta \\ ORCID: https://orcid.org/0000-0002-1489-2009 \\ Universidade Estadual Paulista, Brasil \\ E-mail: luiz.pimenta@unesp.br
}

\begin{abstract}
Resumo
O objetivo da pesquisa foi avaliar o crescimento populacional de Moina sp. alimentadas com quatro dietas por um período de 14 dias. A densidade inicial foi de uma moina adulta/ $\mathrm{L}^{-1}$ de água. As moinas foram cultivadas em 20 recipientes plásticos transparentes com volume útil de $20 \mathrm{~L}^{-1}$. Foi verificada diariamente a temperatura da água, $\mathrm{pH}$, oxigênio dissolvido e condutividade elétrica. Foram testados quatro tipos de dietas e coletadas diariamente cinco amostras de um litro, de cada tanque de cultivo, onde as mesmas foram filtradas em peneira de $800 \mu$ e os organismos colocados num recipiente de $50 \mathrm{~mL}$ com ajuda de uma pisseta, para contagem total sob estereomicroscópio com aumento de até $25 x$. Os parâmetros populacionais avaliados foram: densidade máxima de organismos; dia de máxima densidade; taxa de crescimento específico; tempo de duplicação e rendimento. Os resultados obtidos foram submetidos ao teste $\mathrm{F}$ para análise de variância e análises de regressão linear com o auxílio do pacote estatístico SAS®. Os parâmetros físico-químicos de temperatura, pH, oxigênio dissolvido e condutividade elétrica, se mantiveram dentro do intervalo recomendado para o cultivo. Entretanto, para os parâmetros populacionais de densidade máxima (dieta 4) e taxa de crescimento populacional (dieta 3), apresentaram diferença estatística em relação as outras dietas avaliadas. O parâmetro Tempo de duplicação (Td) não apresentou diferença significativa entre as dietas avaliadas. A dieta mais indicada para o cultivo de Moina sp. foi o tratamento 4 que continha em sua composição Microalga Scenedesmus subspicatus, ração para peixe fermentada, Aptamil premium e Levedura (Saccharomyces cerevisiae).
\end{abstract}

Palavras-chave: Cladocera; Cultivo; Piscicultura ornamental.

\begin{abstract}
The aim of the research was to evaluate the population growth of Moina sp. fed four diets for a period of 14 days. The initial density was one adult mill/ $/ \mathrm{L}^{-1}$ of water. The mills were cultivated in 20 transparent plastic containers with a useful volume of $20 \mathrm{~L}^{-1}$. The water temperature, $\mathrm{pH}$, dissolved oxygen and electrical conductivity were checked daily. Four types of diets were tested and five samples of one liter were collected daily from each culture tank, where they were filtered through an $800 \mu$ sieve and the organisms placed in a $50 \mathrm{~mL}$ container with the help of a pipette, for total counting under a stereomicroscope with magnification of up to $25 \mathrm{x}$. The population parameters evaluated were: maximum density of organisms; day of maximum density; specific growth rate; doubling time and yield. The results were submitted to the F test for analysis of variance and linear regression analysis with the aid of the SAS® statistical package. The physical-chemical parameters of temperature, $\mathrm{pH}$, dissolved oxygen and electrical conductivity remained within the recommended range for cultivation. However, for the population parameters of maximum density
\end{abstract}


(diet 4) and population growth rate (diet 3), they presented statistical difference in relation to the other evaluated diets. The parameter Doubling time (Td) showed no significant difference between the evaluated diets. The most suitable diet for the cultivation of Moina sp. was treatment 4 which contained in its composition Microalgae Scenedesmus subspicatus, fermented fish feed, Aptamil premium and Yeast (Saccharomyces cerevisiae).

Keywords: Cladocera; Cultivation; Ornamental fish farming.

\section{Resumen}

El objetivo de la investigación fue evaluar el crecimiento poblacional de Moina sp. alimentado con cuatro dietas durante un período de 14 días. La densidad inicial fue de un molino adulto / $\mathrm{L}^{-1}$ de agua. Los molinos se cultivaron en 20 recipientes de plástico transparente con un volumen útil de $20 \mathrm{~L}^{-1}$. La temperatura del agua, el pH, el oxígeno disuelto y la conductividad eléctrica se controlaron diariamente. Se probaron cuatro tipos de dietas y se recolectaron cinco muestras de un litro diariamente de cada tanque de cultivo, donde se filtraron a través de un tamiz de $800 \mu$ y los organismos se colocaron en un recipiente de $50 \mathrm{~mL}$ con la ayuda de una pipeta, para el recuento total bajo un estereomicroscopio con aumento de hasta $25 x$. Los parámetros poblacionales evaluados fueron: densidad máxima de organismos; día de máxima densidad; tasa de crecimiento específica; duplicar el tiempo y el rendimiento. Los resultados fueron sometidos a la prueba $\mathrm{F}$ para análisis de varianza y análisis de regresión lineal con la ayuda del paquete estadístico $\mathrm{SAS} \circledast$. Los parámetros físico-químicos de temperatura, $\mathrm{pH}$, oxígeno disuelto y conductividad eléctrica se mantuvieron dentro del rango recomendado para el cultivo. Sin embargo, para los parámetros poblacionales de densidad máxima (dieta 4) y tasa de crecimiento poblacional (dieta 3), presentaron diferencia estadística en relación con las otras dietas evaluadas. El parámetro Tiempo de duplicación (Td) no mostró diferencia significativa entre las dietas evaluadas. La dieta más adecuada para el cultivo de Moina sp. Fue el tratamiento 4 que contenía en su composición Microalgas Scenedesmus subspicatus, pienso fermentado para peces, Aptamil premium y Levadura (Saccharomyces cerevisiae).

Palabras clave: Cladocera; Cultivo; Piscicultura ornamental.

\section{Introdução}

As classes Cladocera e Copepoda se destacam dentro do grupo Crustácea, por serem muito importantes na alimentação da ictiofauna de água doce (Sipaúba-Tavares \& Rocha, 2003). As moinas são microcrustáceos da classe Cladocera muito utilizados como alimento vivo na larvicultura de organismos aquáticos de água doce e principalmente aquariofilia, formando parte do zooplâncton de água doce e tendo importância principal como alimento natural de uma diversidade de larvas de peixes e crustáceos.

No entanto, não existe uma visão clara do que seja uma dieta adequada para moinas usadas como alimento vivo na larvicultura. E atualmente, mesmo com o avanço das pesquisas científicas na área da aquicultura, não existem tecnologias específicas para o cultivo de zooplânctons, que permita reduzir os custos de produção (Muñoz et al., 2013). Somente experimentos de crescimento populacional podem dar a informação mais precisa e isenta de outras influências presentes no meio ambiente.

Neste sentido, é de extrema importância, pesquisas que tenham por objetivo desenvolver técnicas para cultivo em massa de plâncton utilizados na alimentação direta das larvas de peixes ou indiretamente através da alimentação de diversas espécies zooplanctônicas, que por sua vez, constituirão o alimento natural em um sistema de piscicultura (Sipaúba-Tavares \& Bachion, 2002)

Vale lembrar que organismos zooplanctônicos apresentam preferência alimentar por determinadas espécies de microalgas e, dentre os diferentes grupos, os mais adequados seriam as clorofíceas (Scenedesmus subspicatus e Clorella sp) para o cultivo em larga escala de zooplâncton, por apresentarem paredes celulares mais finas e, uma quantidade elevada de carbono orgânico total em relação ao peso seco (Prieto, 2001; Prieto et al., 2006; Sipaúba \& Rocha, 2003) e leveduras (Saccharomyces cerevisiae) (Blanco \& Tacon, 1989; Hoff, 1999; Muñoz et al., 2013).

Os parâmetros demográficos, tais como sobrevivência e reprodução são menos usados em pesquisas, apesar deles serem potencialmente mais valiosos, pois refletem a habilidade do alimento em manter o crescimento populacional do consumidor por um longo tempo (Macedo \& Pinto Coelho, 2000). Entretanto não somente as algas constituem fonte de 
alimento para o zooplâncton, que também utilizam como alimento as bactérias e leveduras (saccharomyces cerevisiae) (Geller \& Muller, 1981) e partículas de matéria orgânica dissolvidas na água.

De acordo com Bachion (1996) e Gorini (2014) o estudo da nutrição de organismos planctônicos em laboratório fornece subsídios para produção em larga escala. A qualidade nutricional do zooplâncton depende principalmente da espécie e de sua alimentação prévia (Prieto \& Atencio, 2008; Naz \& Turkmen, 2009). O conhecimento da biologia e influência de fatores como temperatura e tipo de alimento sobre o crescimento populacional e valor nutricional de suas dietas, permite a manipulação das condições de cultivo, garantindo o sucesso das culturas.

Segundo Sipaúba-Tavares e Bachion (2002) a necessidade do alimento natural para os peixes no início do desenvolvimento pode ser resolvida pelo cultivo de plâncton em instalações especiais designadas para este propósito. Esta é, sem dúvida, a linha a ser seguida, onde a proteína essencial à sobrevivência da larva de peixe poderá ser produzida em massa, em tempo mais curto e em área relativamente menor.

A produção de organismos zooplanctônicos em larga escala consiste em um dos entraves ao desenvolvimento da larvicultura de peixes nativos (Sampaio Nakauth et al., 2015). Portanto, pesquisas relacionadas à alimentação e nutrição de Moina sp. para serem usadas como alimento vivo na larvicultura e aquariofilia são muito importantes para melhorar a produção de larvas e alevinos de peixes nativos e ornamentais. Assim, o objetivo da pesquisa foi avaliar o crescimento populacional de Moina sp. alimentadas com quatro dietas diferentes por um período de 14 dias.

\section{Metodologia}

O experimento foi realizado no Setor de Piscicultura do Campus Professora Cinobelina Elvas, da Universidade Federal do Piauí (CPCE/UFPI), localizado no município de Bom Jesus, estado do Piauí (PI). As moinas foram obtidas a partir de cepas isoladas dos viveiros do setor de piscicultura e foram cultivadas em 20 recipientes plásticos transparentes, cilindro cônico com volume útil de $20 \mathrm{~L}^{-1}$, preenchidas com $18 \mathrm{~L}^{-1}$ de água de poço artesiano repousada por uma semana a fotoperíodo natural e temperatura ambiente durante 14 dias.

A qualidade da água foi verificada diariamente, levando em consideração a temperatura $\left({ }^{\circ} \mathrm{C}\right), \mathrm{pH}$, oxigênio dissolvido $\left(\mathrm{mg} / \mathrm{L}^{-1}\right)$ e condutividade elétrica $(\mu \mathrm{S} / \mathrm{cm})$, com auxílio de um oxímetro (YSI, USA) e pHmetro (YSI, USA). A limpeza dos tanques de cultivo foi realizada uma vez por semana com ajuda de um sifão e reposta à água evaporada. As Moinas foram alimentadas com quatro (4) tipos de dietas diferentes (Tabela 1).

Tabela 1. Dietas experimentais.

\begin{tabular}{ll}
\hline Dieta & Ingredientes utilizados \\
\hline Tratamento 1 & $\begin{array}{l}\text { Microalga Scenedesmus subspicatus; } \\
\text { Ração para peixe fermentada. }\end{array}$ \\
Tratamento 2 & $\begin{array}{l}\text { Microalga Scenedesmus subspicatus; } \\
\text { Ração para peixe fermentada; } \\
\text { Aptamil Premium. }\end{array}$ \\
& Microalga Scenedesmus subspicatus; \\
Tratamento 3 & Ração para peixe fermentada; \\
& Levedura (Saccharomyces cerevisiae). \\
& Microalga Scenedesmus subspicatus; \\
Tratamento 4 & Ração para peixe fermentada; \\
& Levedura (Saccharomyces cerevisiae). \\
\end{tabular}

Rações fermentadas acrescidas de complexo "B" (Bayer) composto por B1 (7 mg), B2 (7 mg), B6 (5 mg), B12 (33 mg) e H (1mg). Fonte: Autores. 
A ração fermentada foi preparada a partir de ração comercial em pó 55\% de proteína bruta (Guabi) para larvicultura de peixe, dissolvida em água deionizada e armazenada em garrafa tipo pet. A formulação das dietas seguiram o seguinte padrão:

- Dieta 1: 10 g. de ração em pó dissolvida em um litro de água deionizada;

- Dieta 2: $10 \mathrm{~g}$. de ração em pó mais $1 \mathrm{~g}$. Aptamil Premium dissolvida em um litro de água deionizada;

- Dieta 3: 10 g. de ração em pó mais 1 g. de Levedura (Saccharomyces cerevisiae);

- Dieta 4: 10 g. de ração em pó mais 1 g. de Aptamil Premium mais 1 g. de Levedura (Saccharomyces cerevisiae).

A cada uma das rações fermentadas foi acrescentado complexo "B" (Bayer) composto por: B1 (7 mg), B2 (7 mg), B6 (5 mg), B12 (33 mg) e H (1 mg) de acordo com Sipaúba-Tavares e Rocha (2003), e deixadas sob aeração durante uma semana e logo filtrada em peneira de $80 \mu$. Após realizado o processamento, cada ração fermentada foi condicionada em garrafas pet de um litro dentro de geladeira e usadas na alimentação das moinas. As diferentes rações fermentadas foram fornecidas na quantidade de $10 \mathrm{~mL}^{-1} \mathrm{em}$ cada tanque de cultivo.

A microalga Scenedesmus subspicatus foi obtida a partir de culturas mantidas no setor de piscicultura do Campus Professora Cinobelina Elvas, da Universidade Federal do Piauí (CPCE/UFPI), Bom Jesus, PI. Foi fornecida nos tanques de cultivo das moinas uma vez por dia às $10 \mathrm{~h} 00$ da manhã até atingir uma densidade de 10 x 106 células $/ \mathrm{mL}^{-1}$ no tanque de cultivo das moinas (ficando com uma coloração esverdeada a água do cultivo).

A densidade inicial de cultivo foi de um (1) indivíduo adulto/ $\mathrm{L}^{-1}$ de água. $\mathrm{O}$ dia do inoculo foi considerado dia zero $(=0)$, e as contagens para estimativa da densidade populacional a partir do segundo dia de cultivo.

Foram coletadas diariamente 5 amostras de um litro, de cada tanque de cultivo. As amostras foram filtradas em peneira de $800 \mu$ e os organismos colocados num recipiente de $50 \mathrm{~mL}$ com ajuda de uma pisseta, para contagem total sob estereomicroscópio (Olympus SZ 40) com aumento de até 25x. Os resultados das médias das contagens das 5 amostras de cada tanque foram usados para a avaliação do crescimento populacional das moinas. A água filtrada na peneira foi devolvida aos tanques de cultivo.

Os parâmetros populacionais avaliados foram: densidade máxima de organismos (Dmo); dia de máxima densidade (Dmd); taxa de crescimento específico $(\mathrm{r})=(\ln \mathrm{Df}-\ln \mathrm{Di}) / \mathrm{t}$; capacidade de suporte $(\mathrm{K})=(\ln \mathrm{Df}-\ln \mathrm{Di}) /(\mathrm{tf}-\mathrm{ti})$; velocidade de crescimento $(\mathrm{v})=(\mathrm{Df}-\mathrm{Di}) / \mathrm{t}($ indivíduos/L/dia); rendimento $(\mathrm{R})=(\mathrm{Df} \mathrm{tf}-\mathrm{Di}$ ti)/t (indivíduos/L/dia); tempo de duplicação $(\mathrm{Td})$ $=\ln 2 / \mathrm{K}($ dias$) ;$ produção diária $(\mathrm{Pd})=(\mathrm{Df}-\mathrm{Di}) /(\mathrm{tf}-\mathrm{ff})($ indivíduos $/ \mathrm{L} / \mathrm{dia}) ;$ densidade populacional final $(\mathrm{Df})=$ indivíduos/L e densidade populacional inicial $(\mathrm{Di})=$ indivíduos/L, conforme descrito em trabalhos de Omori \& Ikeda, 1984; Krebs, 1985; González et al. (1988) e Prieto et al. (2006).

O experimento foi conduzido em delineamento inteiramente casualizado (DIC) com quatro (4) tratamentos representados pelas dietas e cinco (5) repetições. Os resultados obtidos foram submetidos ao teste F para análise de variância (ANOVA) e análises de regressão linear com o auxílio dos comandos PROC GLM e REG do pacote estatístico SAS® (SAS Istitute, versão 9.0, 2000).

\section{Resultados e Discussão}

Os resultados das avaliações dos parâmetros físico-químicos da qualidade da água dos tanques de cultivo de Moina sp. alimentadas com diferentes dietas estão descritos na Tabela 2. 
Tabela 2. Médias e desvio padrão ( \pm ) dos parâmetros físico-químicos da qualidade da água nos tanques de cultivo de Moina sp alimentadas com diferentes dietas

\section{Parâmetros da qualidade da água}

\begin{tabular}{lcccc}
\cline { 2 - 4 } Dietas & $\begin{array}{c}\text { Temperatura } \\
\left({ }^{\circ} \mathbf{C}\right)\end{array}$ & $\mathbf{p H}$ & $\begin{array}{c}\text { Oxigênio dissolvido } \\
\left(\mathbf{m g} / \mathbf{L}^{-\mathbf{1}}\right)\end{array}$ & $\begin{array}{c}\text { Condutividade elétrica } \\
(\mathbf{m V} / \mathbf{c m})\end{array}$ \\
\hline Dieta 1 & $25,05 \pm 1,17$ & $7,31 \pm 0,37$ & $4,53 \pm 0,64$ & $41,03 \pm 11,60$ \\
Dieta 2 & $25,07 \pm 1,07$ & $7,19 \pm 0,39$ & $4,46 \pm 0,58$ & $42,89 \pm 12,43$ \\
Dieta 3 & $25,32 \pm 1,22$ & $7,39 \pm 0,40$ & $4,38 \pm 0,63$ & $45,66 \pm 15,15$ \\
Dieta 4 & $25,31 \pm 1,30$ & $7,32 \pm 0,41$ & $4,36 \pm 0,66$ & $48,97 \pm 17,10$ \\
\hline
\end{tabular}

Fonte: Autores.

Os parâmetros físico-químicos de temperatura, pH, oxigênio dissolvido e condutividade elétrica, se mantiveram dentro do intervalo recomendado para o cultivo de Moina sp. (Romero, 2009). Os valores de temperatura da água se mantiveram constante em todos os tratamentos durante os dias decorrentes do experimento como pode ser observado na (Tabela 2). Tendo em vista que a temperatura da água é um fator limitante na vida do zooplâncton, o possível aumento da temperatura da água acelera o metabolismo dos organismos zooplanctônicos, ocasionando um rápido crescimento, maturidade sexual e aumento da taxa reprodutiva (Melão, 1999; Sarma et al., 2002).

Hoff e Snell (1997) e Rottmann et al. (2003), salientam que a Moina sp. é tolerante a condições extremas de variações diárias de temperaturas $\left(5\right.$ a $\left.31^{\circ} \mathrm{C}\right)$, e apontam como condições ótimas de crescimento temperaturas em torno de 24 a $31{ }^{\circ} \mathrm{C}$. Esta ampla tolerância às variações é uma característica vantajosa para piscicultores comerciais de regiões de tropicais.

Os valores do $\mathrm{pH}$ observados se mantiveram neutros em todas as dietas entre 7,19 \pm 0,39 e máxima de 7,39 $\pm 0,40$ o que se coincide com os valores observados por Velasques et al. (1986); Rojas et al. (2001) e Romero (2009) que observaram um ótimo desenvolvimento de cladócera em ambientes com pH entre 5 e 9.

O oxigênio dissolvido observado se manteve constante em todas as dietas durante o experimento com valores entre 4,36 $\pm 0,66$ de mínima na dieta 4 e máxima de 4,53 $\pm 0,64$ na dieta 1 .

Em relação a condutividade da água observada obteve-se um aumento dos valores relacionados às dietas, com valor mínimo de 41,03 $\pm 11,60$ na dieta 1 e 48,97 $\pm 17,10$ com valor de máxima condutividade na dieta 4 . Esses valores se coincidem ao valor médio encontrado no trabalho de Nakauth et al. (2015) onde observaram valores na faixa de $47,0 \mu \mathrm{s} / \mathrm{cm}^{2} \mathrm{e}$ 63,1 $\mu \mathrm{s} / \mathrm{cm}^{2}$. Esta tendência crescente nos valores de condutividade também foi observada por Macedo (1999), e está relacionada à adição da dieta ao longo do cultivo.

Na Tabela 3 estão apresentados os valores do dia de máxima densidade (Dmd), densidade máxima de organismos (Dmo), densidade inicial organismos por litro de água (Di) de Moina sp. alimentadas com quatro dietas.

Tabela 3. Dia de máxima densidade (Dmd), Densidade máxima de organismos (Dmo), Densidade inicial organismos por litro de água Di (org/ $\left.\mathrm{L}^{-1}\right)$ de Moina sp. alimentada com diferentes dietas.

\begin{tabular}{lcccc}
\hline Parâmetros & Dieta 1 & Dieta 2 & Dieta 3 & Dieta 4 \\
\hline Dmd & $11^{\circ}$ & $11^{\circ}$ & $11^{\circ}$ & $13^{\circ}$ \\
Dmo $\left(\right.$ org $\left./ \mathrm{L}^{-1}\right)$ & 152 & 160 & 186 & 309 \\
Di $\left(\right.$ org $\left./ \mathrm{L}^{-1}\right)$ & 1 & 1 & 1 & 1 \\
\hline
\end{tabular}

Dia de máxima densidade (Dmd), Densidade máxima de organismos (Dmo), Densidade inicial organismos por litro de água Di (org/L ${ }^{-1}$ ), Organismo por litro $\left(\right.$ org $\left./ \mathrm{L}^{-1}\right)$. Fonte: Autores. 
No experimento realizado, em seu décimo terceiro dia apresentou a densidade máxima de 309 organismos a partir do inoculo inicial de um (1) indivíduos adultos $/ \mathrm{L}^{-1}$, onde foi fornecida a dieta 4 com a Microalga Scenedesmus subspicatus acrescida da ração para peixe fermentada, Aptamil premium e Levedura (Saccharomyces cerevisiae).

A menor densidade observada foi obtida na dieta 1 com Microalga Scenedesmus subspicatus mais ração para peixe fermentada. De acordo com (Burian et al.,2014) a qualidade e a quantidade alimentar influenciam aspectos como reprodução, crescimento corporal e populacional e da sobrevivência da comunidade zooplanctônica. A quantidade do alimento fornecido e a qualidade podem ter sido o principal fator que influenciaram a reprodução desses organismos nas dietas 1, 2 e 3 . De maneira geral pode se dizer que dietas com mais suplementos foram mais aceitas pelos organismos, o que refletiu em seu crescimento populacional (Gráfico 1).

Gráfico 1. Curva de crescimento populacional de Moina sp. alimentadas com diferentes dietas.

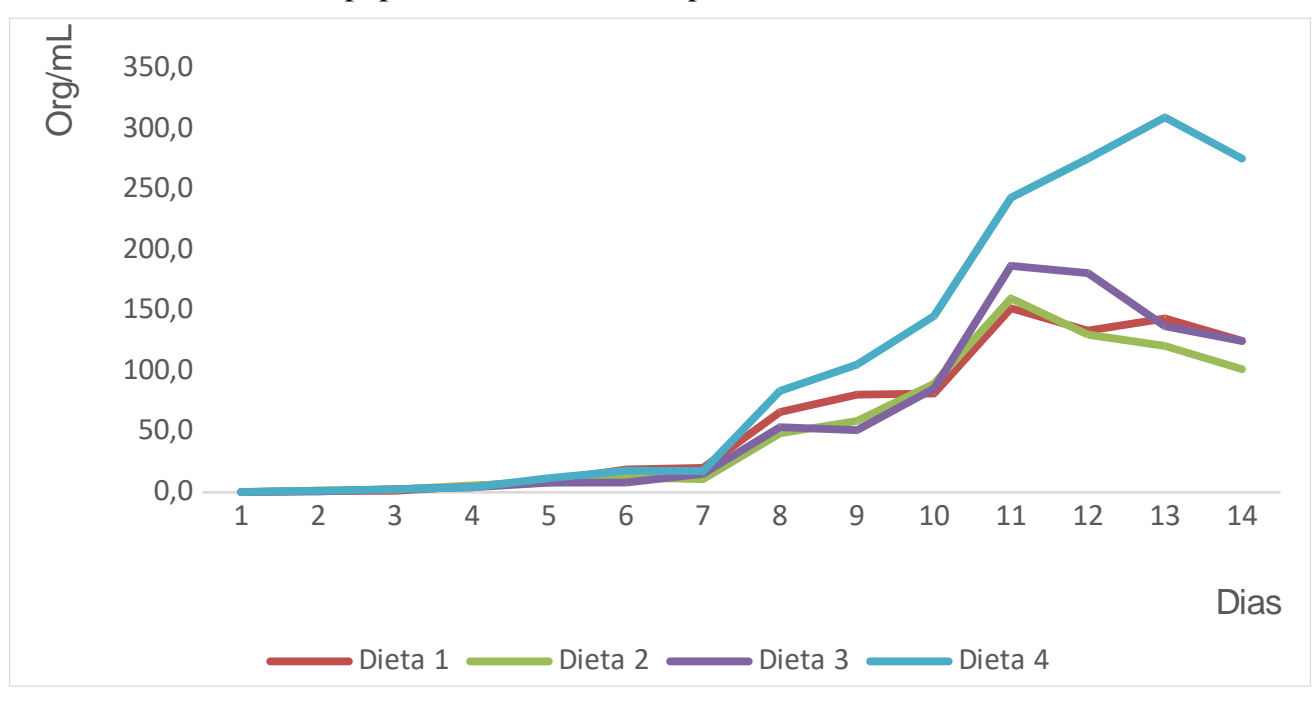

Fonte: Autores.

A curva do crescimento populacional tornou-se constante a partir do $7^{\circ}$ dia de cultivo, quando a densidade de organismos observada foi de 39,5 $\pm 3,41 \mathrm{org} / \mathrm{L}^{-1}$, aumentando progressivamente no $13^{\circ}$ dia quando a densidade atingiu $309,56 \pm$ $5,22 \mathrm{org} / \mathrm{L}^{-1}$ na dieta 4.

Na Tabela 4 estão apresentados os valores médios e desvio padrão ( \pm ) dos parâmetros de crescimento populacional de Moina sp. Cultivada com diferentes dietas, em tanques de $18 \mathrm{~L}^{-1}$ ao longo de 14 dias.

Tabela 4. Medias e desvio padrão ( \pm ) dos parâmetros de crescimento populacional de Moina sp. Cultivada com 4 dietas, em tanques de18 L $\mathrm{L}^{-1}$ ao longo de 14 dias. Densidade máxima (Dmo); Taxa de crescimento populacional (K); Tempo de duplicação (Td) e Rendimento (r).

\begin{tabular}{lcccc}
\hline Parâmetros & Dieta 1 & Dieta 2 & Dieta 3 & Dieta 4 \\
\hline Dmo (org./L $\left.{ }^{-1}\right)$ & $151,96 \pm 50,52 \mathrm{~b}$ & $160,04 \pm 53,75 \mathrm{~b}$ & $186,92 \pm 30,90 \mathrm{~b}$ & $309,56 \pm 5,22 \mathrm{a}$ \\
$\mathrm{K}$ & $0,46 \pm 0,03 \mathrm{a}$ & $0,45 \pm 0,05 \mathrm{a}$ & $0,25 \pm 0,01 \mathrm{~b}$ & $0,44 \pm 0,00 \mathrm{a}$ \\
Td (dias) & $1,49 \pm 0,10 \mathrm{a}$ & $1,56 \pm 0,18 \mathrm{a}$ & $2,78 \pm 0,09 \mathrm{a}$ & $1,57 \pm 0,02 \mathrm{a}$ \\
$\mathrm{r}$ (org/L $\left.\mathrm{L}^{-1}\right)$ & $13,72 \pm 4,59 \mathrm{~b}$ & $14,46 \pm 4,89 \mathrm{~b}$ & $16,90 \pm 2,81 \mathrm{bc}$ & $23,74 \pm 0,40 \mathrm{ac}$ \\
\hline
\end{tabular}

Densidade máxima (Dmo); Taxa de crescimento populacional (K); Tempo de duplicação (Td) e Rendimento (r). Letras diferentes nas filas indicam diferença significativa $(\mathrm{p}<0,05)$ no teste de Tukey. Fonte: Autores. 
Com relação a densidade máxima (Dmo) da população de Moina sp., as dietas 1, 2 e 3 não se diferenciaram estatisticamente, sendo a população de moinas com densidade máxima de 309,56 $\pm 5,22 \mathrm{org} / \mathrm{L}^{-1}$ daquelas alimentadas com a dieta 4 (Microalga Scenedesmus subspicatus mais ração para peixe fermentada mais Aptamil premium mais Levedura (Saccharomyces cerevisiae)).

Romero (2009) obteve como densidade máxima de Moina sp. 9.000 org. $\mathrm{L}^{-1}$ no $7^{\circ}$ a partir de inóculo inicial de 3.000 org. $L^{-1}$. Cornélio (2012) cultivou o mesmo gênero e observou a máxima densidade no $12^{\circ}$ dia, com 9.055 org.L. $L^{-1}$ a partir de inóculo inicial de $6.600 \mathrm{org}$. $\mathrm{L}^{-1}$. Macedo (1999) utilizou duas microalgas na dieta e obteve maiores densidades nas populações alimentadas com Scenedesmus sp. E isso pode ter influenciado essas maiores densidades.

Abrantes et al. (2006) afirmaram que a baixa concentração de nutrientes pode influenciar na produtividade primária, tendo como consequência uma diminuição da biomassa e densidade do fitoplâncton. Por outro lado, um aumento dos nutrientes pode ocasionar um aumento na biomassa fitoplanctônica. Apesar de não ter sido observada correlação significativa dos pigmentos totais em nenhuma das espécies, sabe-se que as altas concentrações de nutrientes e de material em suspensão podem favorecer a proliferação do fitoplâncton (Henry et al., 2006).

A taxa de crescimento populacional (K), não apresentou diferenças significativas entre si nas dietas 1,2 e 4 , mas apresentou diferença significativa na dieta 3 , com a menor taxa de crescimento populacional $0,25 \pm 0,01 \mathrm{org} / \mathrm{L}^{-1}$. Prieto et al. (2006) observou taxas de crescimento de 0,36 \pm 0,002 dia $^{-1}$ e tempo de duplicação de 1,94 \pm 0,012 dias em Moina sp. alimentadas com a mistura da microalga Ankistrodesmus sp. e Saccharomyces cereviseae.

Nakauth et al. (2015) encontraram taxa de crescimento específico de 1,09. Cultivando Moina sp. em sistema estático, alimentadas com microalga Chlorella sp. $\left(1,5 \times 10^{-6} \mathrm{cél}^{\mathrm{m}} \mathrm{ml}^{-1}\right)+$ farinha de peixe $(70 \mathrm{~g})$, resultados menores aos nesse trabalho, isto por que são espécies e metodologias diferentes. Macedo (1999), registrou taxas de crescimento intrínseco de Moina alimentadas com as microalgas Scenedesmus sp. e Ankistrodesmus sp., 0,21.dia ${ }^{-1}$ e 0,15.dia ${ }^{-1}$, respectivamente. Prieto (2006), registrou taxas de crescimento de 0,36 $\pm 0,002$ dia $^{-1}$ e tempo de duplicação 1,94 0,012 dias em Moina sp. alimentadas com a mistura da microalga Ankistrodesmus sp. e Saccharomyces cereviseae.

Em relação ao tempo de duplicação $(\mathrm{Td})$ não houve diferenças significativas $(\mathrm{p}<0.05)$ entre as diferentes dietas. Prieto et al. (2006) pesquisando parâmetros populacionais de Moina sp. alimentadas com Ankistrodesmus sp. mais Saccharomyces cereviseae na proporção 1:1, sendo a mais adequada para o cultivo de moina com um tempo de duplicação de $1,94 \mathrm{e}$ apresentando um valor semelhante ao encontrado nesse trabalho, podendo ser que se trata de outra espécie de Cladócera.

No rendimento ( $r)$ não houve diferença significativa $(p<0,05)$ entre as dietas 1,2 e 3 e estas apresentaram diferenças significativas $(\mathrm{p}<0,05)$ entre a dieta 4 na qual se obteve o maior rendimento com $23,74 \pm 0,40 \mathrm{org} / \mathrm{L}^{-1}$ e o menor rendimento se obteve na dieta 1 com $13,72 \pm 4,59$ org $/ \mathrm{L}^{-1}$.

O rendimento da cultura de Cladocera e Copepoda, apesar de inferiores aos de Rotífera e Ciliophora são relevantes como fonte de alimentos para larvas e alevinos. Estes itens alimentares são indispensáveis na dieta das diferentes espécies de peixes porque constituem presas de mais fácil captura nos estágios em que a predação é eminentemente visual (Zaret, 1980).

\section{Conclusão}

A dieta referente ao tratamento 4 contendo Microalga Scenedesmus subspicatus, ração para peixe fermentada, Aptamil Premium e Levedura (Saccharomyces cerevisiae) foi a que mostrou resultados mais satisfatórios nas condições testadas para o cultivo de Moina sp. 


\section{Referências}

Abrantes, N., Antunes, S. C., Pereira, M. J., \& Gonçalves, F. (2006). Seasonal succession of cladocerans and phytoplankton and their interactions in a shallow eutrophic lake (Lake Vela, Portugal). Acta Oecologica. 29: 54-64.

Bachion, M. A. (1996). Estudo do crescimento e desenvolvimento populacional de três espécies zooplanctônicas submetidos a diferentes dietas alimentares. Dissertação. Centro de Aqüicultura da Universidade Estadual Paulista Campus de Jaboticabal - SP. 146p.

Blanco, L., \& Tacon, A. (1989). La producción de alimento vivo y su importancia en acuacultura una diagnosis. FAO-Itália, 81p.

Burian, A., Kainz, M. J., Schagerl, M., \& Yasindi, A. (2014). Species-specific separation of lake plankton reveals divergent food assimilation patterns in rotifers. Freshwater biology, 59(6), 1257-1265.

Cornélio, J. P. D. S. (2012). Isolamento e produção de Chlorella sp. (Chlorophyceae) e Moina sp. (Cladocera) para utilização na larvicultura de Matrinxã (Brycon amazonicus). Dissertação. Universidade Nilton Lins, Manaus.

Geller, W., \& Muller, H. (1981). The filtration apparatus of Cladocera: Filter mesh-sizes and their implementations food selectivity. Acta Oecologia. 49, 316321.

González, A. (1988). El Plancton de las águas continentales. Secretaria general de la O.E.A. Serie de Biología; Monografía. 33 Washington D.C.

Gorini, T. L. A. (2014). Crescimento populacional, qualidade nutricional e eficiência fotoquímica de microalgas continentais cultivadas em diferentes espectros de luz e disponibilidades de N-P-K. Campos dos Goytacazes. Tese (Doutorado). Universidade Estadual do Norte Fluminense Darcy Ribeiro.

Henry, R., Ushinohama, E., \& Ferreira, R. M. R. (2006). O fitoplâncton em três lagoas e no Rio Paranapanema na zona de sua desembocadura na Represa de Jurumirim durante um período prolongado de seca. Revista Brasileira de Botânica. 29(3), 399-414.

Hoff, H. F., \& Snell, W. T. (1999). Plankton Culture Manual, Florida Aqua Farms, Inc. 160p.

Hoff, F., \& Snell, T. (1997). Plankton culture manual. Florida Aqua Farms, Inc. Florida (USA), p. 108-122.

Krebs, C. J. (1985). Ecology: the experimental analysis of distribution and abundance. New york: Harper Collins.

Macedo, C. F. (1999). O estudo da qualidade nutricional de duas espécies de cladóceros em relação às clorofíceas Ankistrodesmus gracilis e Scenedesmus quadricauda. Dissertação. Universidade Federal de Minas, Belo Horizonte, Minas Gerais.

Macedo, C. F., \& Pinto-Coelho, R. M. (2000). Efeito das algas Ankistrodesmus gracilis e Scenedesmus quadricauda no crescimento e no índice lipídico de Daphnia laevis e Moina micrura. Acta Scientiarum. Biological Sciences, 22, 397-401.

Melão, M. G. G. (1999). Desenvolvimento e aspectos reprodutivos de cladóceros e copépodos de águas continentais brasileiras. In: Pompeo, M. L. M. (ed.). Perspectivas da Limnologia no Brasil. Gráfica e Editora União: São Luís, 45-57.

Muñoz, M., Medina, V. M., \& Cruz Casallas, P. E. (2013). Efecto del fotoperiodo y del alimento sobre la productividad de dos cladóceros nativos (Moina sp. y Diaphanosoma sp.) de la Orinoquia colombiana. Revista U.D.C.A. Actualidad \& Divulgación Científica, 16, $167-174$.

Nakauth, A. C. S. S., Müller, R. L., Villacorta-Correa, M. A., Acioli, A. N. S., \& de Almeida, R. (2015). Crescimento Populacional do Cladocera Moina Sp. em Sistema De Cultivo Estático. Aninc-Anuário do Instituto de Natureza e Cultura, 1, 18-29.

Naz, M., \& Türkmen, M. (2009). Changes in the digestive enzymes and hormones of gilthead seabream larvae (Sparus aurata, L. 1758) fed on Artemia nauplii enriched with free lysine. Aquaculture international, 17(6), 523-535.

Omori, M., \& Ikeda, T. (1984). Methods in Marine Zooplankton Ecology, xiii, John Wiley, 1984.332.

Prieto, M. (2001). Aspectos reproductivos del cladócero Moinodaphnia sp en condiciones de laboratorio. Revista Medicina Veterinaria y Zootecnia, 6, 102110.

Prieto, M., De La Cruz, L., \& Morales, M. (2006). Cultivo experimental del cladócero Moina sp alimentado con Ankistrodesmus sp y Saccharomyces cereviseae. Revista Medicina Veterinaria y Zootecnia, 11, 705-714.

Prieto, M., \& Atencio, V. (2008). Zooplancton en la larvicultura de peces neotropicales. Revista Medicina Veterinaria y Zootecnia, 13 , $1415-1425$.

Rojas, N. E. T., Marins, M. A., \& Rocha, O. (2001). The effect of abiotic factors on the hatching of Moina micrura Kurz, 1874 (Crustacea: Cladocera) ephippial eggs. Brazilian Journal of Biology, 61(3), 371-376.

Romero, L. T. (2009). Desarrolo de Moina sp. em condiciones de laboratório alimentado com microalgas cultivadas en residuales pesqueiros.(Development of Moina sp. in laboratory conditiones, fed with microalgae cultured in the waste waters of fishing industry). Revista Eletrônica Veterinária. 10(4).

Rottmann, R. W., Graves, J. S., Watson, C., \& Yanong, R. E. (2003). Culture Techniques of Moina: The ideal Daphnia for feeding freshwater fish fry. Department of Fisheries and Aquatic Sciences, Florida Cooperative Extension Service, Institute of Food and Agricultural Sciences, University of Florida, Circular 1054.

Sampaio Nakauth, A. C. S., Muller, R. L., Villacorta-Correa, M. A., Acioli, A. N. S., \& Almeida, R. (2015). Crescimento populacional do cladocera Moina sp. em sistema de cultivo estático. Anuário do instituto natureza e cultura/ufam. Disponível em: http://www.periodicos.ufam.edu.br/index.php/ANINC.

Sarma, S. S. S., Nandini, S., \& Gulati, R. D. (2002). Cost of reproduction in selected species of zooplankton (rotifers and cladocerans). Hydrobiologia, 481, 89-99. 
Research, Society and Development, v. 10, n. 13, e73101320909, 2021

(CC BY 4.0) | ISSN 2525-3409 | DOI: http://dx.doi.org/10.33448/rsd-v10i13.20909

Sipaúba-Tavares, L. H., \& Bachion, M. A. (2002). Population growth and development of two species of Cladocera, Moina micrura and Diaphanososma birgei. Brazilian Journal of Biology. 62(4), 701-711.

Sipaúba-Tavares, L. H., \& Rocha, O. (2003). Produção de placton (Fitoplancton e zooplancton) para alimentação dos organismos aquáticos. Editorial São Carlos RiMa. Brasil. 120p.

SAS®. (2002). SAS Istitute, versão 9.0.

Vázquez, A., Solis, E., Macedo, N., \& Rosas, I. (1986). Influencia de la calidad del agua sobre la ocurrencia de Daphnia pulex en la presa José Antonio Alzate y algunos aspectos de su pesquería. Revista Internacional de Contaminación Ambiental, 2(1), 39-56.

Zaret, T. M. (1980). Predation and Freshwater Comunnites. Yale Univ. Press, London.187p. 